Essay Review

\title{
Gewalt in der Frühen Neuzeit - Positionen der Forschung
}

Maike Christadler

Keywords: violence; body; honour; mediality; perception; representation; art; early modern period

Gewalt ist in den letzten zwei Jahrzehnten zu einem dominanten Thema in zahlreichen wissenschaftlichen Disziplinen geworden, von der Geschichte über die Soziologie und Politikwissenschaft zu den Literatur- und Kulturwissenschaften, in keinem Fach wurde nicht über Gewalt nachgedacht. Und wenn man davon ausgeht, dass Wissenschaft sich innerhalb der Umwelt entwickelt, in der sie angesiedelt ist, so spiegelt sich in dem Thema ein gesamtgesellschaftliches Interesse. Die Terroranschläge des 11. September 2001 und die Kriege, die sie legitimiert haben, der Amoklauf in Erfurt und die angeheizte Diskussion um die Rolle von Gewaltdarstellungen in Computerund Videospielen ${ }^{1}$, die Folterungen von Abu Ghraib und ihre mediale Inszenierung: Gewalt ist ein Teil aller Ebenen unserer Lebenswelt. Bereits in dieser reduzierten Aufzählung wird deutlich, dass Gewalt ein ungeheuer vielschichtiges Phänomen ist, das von Kriegen bis zu Computerspielen reicht und eben auch deshalb in den verschiedensten Disziplinen und mit verschiedensten methodischen Ansätzen diskutiert wird.

1 www.sueddeutsche.de/computer/artikel/268/92176/ und www.sueddeutsche.de/dossiers/dossier/307/91216/.

Dr. Maike Christadler, Historisches Seminar, Universität Basel, Hirschgässlein 21, CH-4051 Basel (maike.christadler@unibas.ch). 


\section{Der Gewaltbegriff in der Forschung}

Für die Forschung stellt sich zunächst die Frage, was unter Gewalt verstanden wird. Gertrud Nunner-Winkler unterscheidet physische, psychische, kulturelle und strukturelle Gewalt ${ }^{2}$. Während erstere relativ leicht als die «zielgerichtete Schädigung von Menschen» definiert werden kann, ist der Einbezug von verbalen Beleidigungen oder Beschimpfungen als psychische Gewalt schon schwieriger einzugrenzen. Kulturelle ${ }^{3}$ und strukturelle ${ }^{4}$ Gewalt bezeichnen auch Zwänge, die keine körperliche Dimension haben und meist von Institutionen ausgeübt werden. In diese Richtung geht auch die Formulierung von Pierre Bourdieu, der von «symbolischer Gewalt» spricht und damit eine Anerkennung der Autorität durch die Beherrschten meint, die nicht freiwillig erfolgt, sondern «abgepresst» ist - mangels Möglichkeit, sich dagegen zu entscheiden ${ }^{5}$. Schon die Kulturarbeit des Einschliessens und Ausschliessens, also des generellen Kategorisierens und Klassifizierens, enthält aufgrund ihrer wertenden Zuordnungen eine Gewaltsamkeit. Insgesamt ist mit diesem erweiterten Gewaltbegriff bereits das Schädigen einer Person durch unterlassene Hilfeleistung - auch bezogen auf die Duldung sozial unterprivilegierter Lebensverhältnisse - ein Akt der Gewalt. Diese Ausweitung des Verständnisses von Gewalt hat interessante Fragestellungen und Ergebnisse hervorgebracht, aber auch fundamentale Kritik provoziert: Die inflationäre Verwendung des Begriffs verharmlose letztlich die tatsächliche (physische) Gewalttat, die analytisch nicht mehr fassbar sei ${ }^{6}$.

Diese Auffassung führt dazu, dass ein Grossteil der - zumindest deutschsprachigen - Forschung zur Frühen Neuzeit sich heute vornehmlich eines Gewaltbegriffs bedient, der «auf einer am Körperlichen zumindest orientierten Beziehung zwischen einer die Gewalt hervorbringenden Ursache auf der einen und der daraus ableitbaren, durch die Betroffenen erfahrenen (erlittenen) Wirkung auf der anderen Seite» basiert ${ }^{7}$. Damit ist Gewalt zunächst als ein Angriff auf körperliche Integrität definiert, die von Personen ausgeübt und von anderen Personen erfahren wird. Mit dieser strikteren Definition von Gewalt werden jedoch Machtverhältnisse, die institutionell, sozial oder aufgrund von Geschlechtszugehörigkeit gegeben sein können, ebensowenig fassbar wie die psychische Gewalterfahrung. Ausserdem suggeriert die Beschränkung der Analyse auf physische Gewaltakte, dass eine

\footnotetext{
2 Nunner-Winkler 2004.

3 Bauman 1995.

4 Galtung 1975.

5 Bourdieu 1990. Vgl. auch Nolde 2003, 6f.

6 Sieferle/Breuninger 1998; Nunner-Winkler 2004.

7 Mommertz 2005, 343.
} 
körperliche Erfahrung direkt zugänglich zu machen sei. Jedoch ist auch körperliche Gewalt immer medial gebrochen: Im Mittelalter und in der Frühen Neuzeit sind es schriftliche oder bildliche Quellen, die Gewalt darstellen und gemäss ihrer spezifischen rhetorischen, literarischen, juristischen oder eben ikonographischen Traditionen und Modelle formen. Doch selbst die aktuelle Gewalt - jedenfalls in der ziemlich befriedeten Kultur Westeuropas - ist der medialen Vermittlung unterworfen, sei es durch Fernsehnachrichten oder durch Hollywoods Bilderindustrie. An die «tatsächliche» Gewalt kommen wir nicht heran, wir müssen ihre rhetorische und visuelle Verarbeitung stets mit bedenken.

\section{Historische Gewaltforschung}

Das neuzeitliche Geschichtsdenken hat seit dem 18. Jahrhundert eine Selbstbeschreibung der westlichen Gesellschaften hervorgebracht, nach der diese in einem Prozess der «Zivilisierung» begriffen seien, der schliesslich zur Überwindung von Gewalt und zur Herrschaft von Vernunft und Recht führen würde ${ }^{8}$. Mit seinem einflussreichen Buch Über den Prozeß der Zivilisation hat Norbert Elias dieses Narrativ der steten «Verbesserung» der menschlichen Gesellschaft massgeblich geprägt ${ }^{9}$. «Zivilisierung» bedeutet nach Elias eine Monopolisierung der Gewaltausübung beim Staat und eine zunehmende Affektbeherrschung aufgrund einer Internalisierung von Zwängen im Individuum. Eine solche teleologische Entwicklungsgeschichte muss ihre Anfänge in eine Zeit setzen, die sie selbst als ihr «Anderes» versteht. So wurden - vor allem in den Lektüren von Elias - das Mittelalter und auch noch die Frühe Neuzeit zu «Gegenbildern» unserer modernen Zeit: urwüchsiger, kraftvoller, spontaner, aber eben auch grausam, gewalttätig und unzivilisiert. Doch sowohl dieses Modell eines linearen Fortschritts als auch die mit ihm verbundenen Bilder vom «düsteren Mittelalter» sind grundlegend kritisiert und von Ansätzen abgelöst worden, die nicht die longue durée anvisieren, sondern nach historisch spezifischen Funktionen von Gewalt fragen ${ }^{10}$.

Diese Kritik richtete sich auch gegen eine traditionelle Geschichtswissenschaft, für die lange Zeit die Prozesse von Staatsbildung und die Konstitution von Herrschaft (und damit häufig Kriege und Kriegführung) im Zentrum der Aufmerksamkeit standen. Gewalt herrschte dort, wo (politische) Interessen

8 Sieferle/Breuninger 1998, 22-24.

9 Elias 1939.

10 Dinges 1998; Schwerhoff 1998. 
durchgesetzt und Widerstand gebrochen werden musste. Der anthropological turn dagegen lenkte die Aufmerksamkeit der Geschichtswissenschaft auf die handelnden Personen in ihrer Alltagswelt und machte die (körperliche) Gewalt zu einem einschlägigen Forschungsfeld ${ }^{11}$. In diesen verschiedenen Forschungsinteressen spiegelt sich die Doppeldeutigkeit des Gewaltbegriffs: «Gewalt» kann im Deutschen sowohl potestas als auch violentia bedeuten. Während ersteres stärker an die Begriffe von Macht und Herrschaft gekoppelt ist, wird mit letzterem eher die körperliche Gewalt gefasst ${ }^{12}$. Es ist vor allem die Dimension von violentia, die im Rahmen der historischen Anthropologie ${ }^{13}$ und angrenzender Forschungsbereiche untersucht worden ist. So wird in der Frauen- und Geschlechtergeschichte generell Gewalt gegen und von Frauen ${ }^{14}$, aber auch eheliche Gewalt thematisiert ${ }^{15}$. In einer anthropologisch-feministischen Geschichte der Gynäkologie wurde nach der sozialen und medizinischen Gewalt eines (illegalen) Schwangerschaftsabbruchs gefragt ${ }^{16}$. Die Kriminalitätsforschung im allgemeinen interessiert sich für die sozialen Funktionsmechanismen von Gewalt ${ }^{17}$. Dabei stehen weniger die Ursachen im Zentrum des Interesses als vielmehr die Praktiken im Umgang mit Gewalt, sei es in ihrer Ausübung oder in ihrem Erleiden ${ }^{18}$. Gewalt wird so dezidiert als historische Kategorie konzeptualisiert und nicht als anthropologische Konstante oder menschliches Schicksal betrachtet, wie es Wolfgang Sofsky in seinem Traktat über die Gewalt tut ${ }^{19}$. Gewalt wird gemacht, ihre Wahrnehmung und Wertung sind historischem Wandel unterworfen und kulturell spezifisch ${ }^{20}$.

Gerade für die Forschung zum Mittelalter und der Frühen Neuzeit hat die Kategorie der Gewalt ermöglicht, neue Perspektiven zu eröffnen. Galt Gewalt - wo nicht staatlich legitimiert - in der älteren Forschung meist als ausschliesslich zerstörerisch und unproduktiv, so wird sie in neueren Arbeiten als ordnungsstiftendes Element sichtbar gemacht. Wegweisend für die Untersuchung von «Riten der Gewalt» war die schon in den 1970er Jahren erschienene Aufsatzsammlung von Natalie Zemon Davis zu Gesellschaft und Kultur im frühneuzeitlichen Frankreich. Sie analysiert die grausamen Gewaltausbrüche der Religionskriege als «Reinigungsrituale», die politisch,

11 Traverse 1995; L'Homme 1996.

12 Meumann/Niefanger 1997,11-13.

13 Ulbrich/Jarzebowski/Hohkamp 2005; Historische Anthropologie 2004.

14 Gender \& History 16/3 (2004); Hilbig/Kajatan/Miethe 2002.

15 Chaytor 1995; Nolde 2003.

16 Faro 2002.

17 Burghartz 1990; Schwerhoff 1999.

18 Althoff 1998.

19 Anders Sofsky 1996 und 2002.

20 Ulbrich/Jarzebowski/Hohkamp 2005, 13. 
kulturell und religiös kodierten Verhaltsmustern folgen, die den jeweiligen Feind demütigen und die von ihm ausgehende Bedrohung bannen sollten ${ }^{21}$. Gadi Algazi konnte bezüglich der mittelalterlichen Fehde zeigen, dass sie einerseits ein Instrument der Auseinandersetzung zwischen Adeligen war, aber andererseits vor allem der Regulierung des Verhältnisses zwischen Adeligen und ihren Untertanen diente. In regelmässigen Abständen wurden die Bauern über die Zerschlagung ihres Besitzes «zurechtgestutzt» ${ }^{22}$. Noch in der ausufernden Grausamkeit der Religionskriege im Frankreich des 16. Jahrhunderts, die selbst von den Zeitgenossen kaum begründet werden konnte, weist Denis Crouzet Rituale der Sinnstiftung nach, in denen von katholischer Seite Gewalt als mystische Vereinigung mit Gott und von protestantischer Seite als gottgewolltes Zeichen des Triumphes des Evangeliums erlebt wird ${ }^{23}$. Valentin Groebner zeigt, dass im spätmittelalterlichen Nürnberg körperliche Extremitäten sehr gefährdet sind, denn in den Quellen tauchen sie häufig als abgeschnittene auf. Aber er weist auch nach, dass ein Grossteil der Körperverletzungen mit einer gütlichen Einigung zwischen Täter und Opfer geregelt wird - auf Betreiben der Stadt. Die «privaten» Gewalttätigkeiten wurden so einer städtischen Ordnungs- und Definitionsmacht unterworfen ${ }^{24}$. Das Beispiel macht deutlich, dass potestas und violentia eng miteinander verbunden sind und dass die Ausübung physischer Gewalt in einem Rahmen geschieht, der von politischen, kulturellen, gesellschaftlichen Normen definiert und gesteuert wird. Auch wenn es sinnvoll ist, beide Formen der Gewaltausübung analytisch zunächst zu unterscheiden (Staats-/Herrschaftsgewalt vs. körperliche Gewalt), so müssen beide Formen doch aufeinander bezogen werden, um gesellschaftliche Gewaltphänomene erklärbar zu machen.

Gewalt ist also in der spätmittelalterlichen und frühneuzeitlichen Gesellschaft ein Medium des Aushandelns; ihre Ausübung lässt sich als komplexes Kommunikationssystem lesen, in dem soziale Hierarchien abgesichert, aber auch stets neu festgeschrieben werden. Gewalt läuft keineswegs spontan und unkontrolliert ab, vielmehr folgt sie bestimmten Regeln und Ritualen und ist entsprechend nicht Ausdruck einer unzivilisierten, vormodernen männlichen Triebnatur, sondern das Produkt einer Kultur, die solche gewalthaften Auseinandersetzungen verlangte, formte und regulierte ${ }^{25}$.

21 Zemon Davis 1987.

22 Algazi 1995, 64; Algazi 1996.

23 Crouzet 1995; Crouzet 2005.

24 Groebner 1995.

25 Burghartz 1990. 


\section{Gewalt am Körper}

Gewalt spielt also im frühneuzeitlichen Europa eine entscheidende Rolle bei der Regelung sozialer Beziehungen. Diese werden jedoch zumeist an Körpern ausgetragen. Deshalb sind für die historische Gewaltforschung die Erkenntnisse der Körpergeschichte von zentraler Bedeutung. So wie Gewalt nicht als eine anthropologische Konstante oder gar als menschliches Schicksal verstanden werden kann, so hat auch die Körpergeschichte gezeigt, dass der Körper keine essentielle Unveränderlichkeit ist, sondern historisch geformt wird ${ }^{26}$.

Die Gewaltforschung, die sich mit der Zerstörung oder Verletzung körperlicher Identität beschäftigt, steht immer wieder vor dem Problem, ihren Gegenstand überhaupt erst konstituieren zu müssen, gerade weil Körper nicht problemlos das Refugium eines wie auch immer aufgefassten «Natürlichen» sind: «Körper sind immer, welche Gesellschaft man auch betrachtet, eingebunden in ein spezifisches Geflecht historischer Bedingungen (Praxisformen, Strukturen, Symbolsystem), die eine direkte Annäherung unmöglich machen.» So formuliert Peter Burschel und schliesst daran die Forderung an, danach zu fragen, welchen Platz körperliche Unversehrtheit in der jeweiligen gesellschaftlichen Ordnung habe ${ }^{27}$. Über die Körpergeschichte sind auch die Gewalt- und Schmerzforschung miteinander verbunden. Letztere hat wesentliche Erkenntnisse zur Historisierung von Wahrnehmung und zum Umgang mit der «Unausdrückbarkeit» von Erfahrung geliefert ${ }^{28}$. Sie ist jedoch weniger an den gewalttätigen Ursachen des Schmerzes interessiert, die hier im Zentrum stehen. Mehr als über den individuellen Schmerz, über die Erfahrung, Gewalt ausgesetzt zu sein, sagen die Quellen darüber, mit welchem Ziel und mit welcher symbolischen Bedeutung Körper geschunden, verstümmelt und versehrt werden ${ }^{29}$. Valentin Groebner hat sich immer wieder mit dem Zeichencharakter von Gewalt auseinandergesetzt und verfolgt, wie z.B. der Angriff auf das Gesicht eines Gegenübers - oft als Abschneiden oder Verletzen der Nase ausgeführt - in ein Kommunikationssystem von Ehre und Schande eingebunden ist. In den Nürnberger Gerichtsakten vom ausgehenden 15. Jahrhundert finden sich Ehefrauen, die ihren untreuen Männern oder ihren Nebenbuhlerinnen die Nasen abschnitten, ebenso wie gehörnte Ehemänner, die ihren Nebenbuhlern oder sogar deren (unschuldigen) Frauen dieselbe Verstümmelung zufügten. Damit wurde eine

26 Lorenz 2000.

27 Burschel/Distelrath/Lembke 2000, 17.

28 Scarry 1992; Rey 1993; Morris 1994.

29 Porret 1998. 
symbolische Kastration vollzogen, die zugleich eine körperliche Strafe und eine lebenslange «Zeichnung» war ${ }^{30}$. Deutlich wird an Groebners Ausführungen, dass in der Körperverletzung des Naseabschneidens zahlreiche Diskurse mit aufgerufen werden. Die theologische Frage der (verletzlichen) Gottesebenbildlichkeit des Menschen wird ebenso verhandelt, wie sexuelle Ehre und Ehrbarkeit markiert werden, und literarische Texte finden ihren Widerhall in einer symbolisch aufgeladenen Realität.

Zugleich wird deutlich, dass Gewalt auch geschlechtlich kodiert ist, denn nur eine Frau konnte zu einer offenkundigen Ehebrecherin gemacht werden, indem man ihr die Nase abschnitt, während ein Mann, mit demselben Zeichen markiert, als «Ungestalt» wahrgenommen und mit Homosexualität in Verbindung gebracht wurde ${ }^{31}$.

«Ehre» als zentrale regulatorische Idee hat in den letzten Jahren viel zur Erklärung von Gewalthandlungen beigetragen. Wie in Groebners Beispiel sind Ehre und Geschlecht im Kontext von Gewaltausübung oft eng miteinander verbunden. Meist ist es männliche Gewalt, die über Ehr-Konzepte fassbar wird, und Männer stellen bei weitem die meisten Gewalttäter und auch Opfer. Dieser statistische Befund verleitet dazu, männliche Gewalt als Teil der männlichen Natur wahrzunehmen, die eben so zu akzeptieren $\mathrm{sei}^{32}$. Dagegen ist jedoch auch gezeigt worden, dass Gewalt gerade keine Konstante, sondern ein komplexes, historisch variables gesellschaftliches Konstrukt ist. Erst die Analyse der spezifischen Umstände von Gewalt gestattet Aussagen über ihre Geschlechtsgebundenheit und lässt sie überhaupt als Untersuchungskategorie nützlich werden. So demonstriert Brigitte Rath am Beispiel von Bozen um 1500, wie stark gewalttätige Auseinandersetzungen den Alltag prägten, und stellt einen engen Zusammenhang von männlichem Habitus und physischer Gewalt her ${ }^{33}$; Susanna Burghartz sieht in männlicher Gewaltanwendung sogar «eine zentrale Anforderung des Ehrencodes in Auseinandersetzungen zwischen Männern $»^{34}$. Dennoch ist es gerade nicht eine universell determinierte männliche Physis, die Gewalt hervorbringt. Vielmehr unterlag Gewalt zwischen Männern kulturell festgelegten Regeln, die immer neu gelernt und definiert wurden.

Frauen werden dagegen meist als Opfer von Gewalt greifbar. Neuere Arbeiten dokumentieren zwar nur wenige Tötungsdelikte, sie haben aber eine durchaus heftige Gewalttätigkeit von Frauen sichtbar gemacht, gerade

30 Groebner 2003, hier 77-92.

31 Groebner 2003, 86.

32 Spierenburg 1998.

33 Rath 1996.

34 Burghartz 1990, 200. 
auch im Kontext von Ehrhändeln ${ }^{35}$. Dorothea Nolde hat gezeigt, dass vor dem Pariser Ehegericht im 17. Jahrhundert der Gattenmord zu einem zentralen Parameter in der Neudefinition von Geschlechterrollen in der Ehe wurde ${ }^{36}$.

Trotz dieser Befunde muss die Frage nach der Gewalt von Frauen anders gestellt werden: Auseinandersetzungen zwischen Frauen oder auch zwischen Frauen und Männern wurden nach anderen Regeln geführt als «rein» männliche Gewalt. Männer waren meist zumindest mit Messern bewaffnet, so dass starke Verletzungen oder gar Tötungsdelikte relativ häufig waren. Gerichtsverhandlungen in der Vormoderne machen dagegen deutlich, dass Angriffe auf Personen keineswegs nur physisch, sondern auch verbal erfolgen konnten und dass Beleidigungen und Verleumdungen in ihren sozialen Konsequenzen für die Ehre der Betroffenen ebenso schwer wiegen konnten wie Körperverletzungen und deshalb vor Gericht ebenso wie physische Übergriffe angeklagt und verhandelt wurden. Beleidigungen waren keine «harmlosen» verbalen Attacken, denn die Ehre gerade einer Frau, d.h. ihr ehrbarer Ruf, «war für ihre sozialen Beziehungen und ihr Selbstgefühl von grösster Bedeutung und konnte durch Beleidigungen und Verleumdungen ernsthaft gefährdet oder sogar zerstört werden» ${ }^{37}$. Hier zeigt sich, dass ein zu eng gefasster Gewaltbegriff, der sich ausschliesslich auf eine körperliche Verletzung bezieht, nicht ausreicht, um die vormoderne Wahrnehmung von Gewalt $\mathrm{zu}$ rekonstruieren.

Der in der Aufklärung etablierte Blick auf Geschichte als Fortschrittsgeschichte verstellt noch heute oft die Wahrnehmung historischer Differenz. Historische Ereignisse und Verhältnisse werden meist im Hinblick auf das Heutige als Vorformen oder Vorstufen interpretiert, die «überwunden» worden sind. Teil dieses Fortschrittsnarrativs ist auch die Vorstellung einer Trennung von Körper und Geist: der als sündig und lustbesetzt konzipierte Leib muss durch den Geist - der an der geistigen Welt teilhaben will - reguliert und kontrolliert werden. Doch in der Frühen Neuzeit waren Körper und Seele sehr viel stärker als Einheit konzipiert. Die Psyche wurde vom Leib weniger deutlich getrennt imaginiert, weshalb auch psychische und körperliche Verletzung sich weniger leicht trennen lassen. «Leib und Seele [sind] über sympathetische Beziehungen miteinander verbunden», «menschliches Tun, Prozesse des Naturgeschehens und göttliches Handeln [bilden] einen umfassenden Wirk- und Verweiszusammenhang», so Andreas Bähr ${ }^{38}$. Diesen «Verweiszusammenhang» hat Monika Mommertz kürzlich als «ima-

35 Burghartz 1999.

36 Nolde 2003.

37 Burghartz 1999, 35.

38 Bähr 2005, 363. 
ginative Gewalt» bezeichnet. Sie macht darauf aufmerksam, dass bei einer Beschränkung des Gewaltbegriffs auf körperliche Übergriffe vormoderne Wahrnehmungen von Gewalt ausgeschlossen werden, die nicht das Ergebnis von Tätlichkeiten oder körperlichen Aggressionen sind, sondern als Auswirkungen von Zauberei bezeichnet werden können - Imaginationen, die als extrem real erlebt werden (wie z.B. der Hexenglauben) ${ }^{39}$. Die «imaginative Gewalt» äusserte sich durchaus in körperlichen Symptomen, die als Schmerz und Leid perzipiert wurden. Mommertz plädiert dafür, der gedachten Gewalt einen eigenen Stellenwert einzuräumen, denn sie diente, ebenso wie die körperlichen Rituale gewalttätiger Auseinandersetzungen, der sozialen Kommunikation, mit der gesellschaftliche Hierarchien jeweils neu verhandelt, verfestigt und dynamisiert werden konnten.

Aufgrund dieser Forschungen wird deutlich, dass eine Erweiterung des Gewaltbegriffs nötig ist, um die vormoderne Dimension von «Gewalt» überhaupt adäquat fassen zu können. Die Einbeziehung von verbaler und imaginativer Gewalt leistet in der Analyse eine genauere Differenzierung eines geschlechtsspezifischen Funktionierens von Gewalt und umfasst auch solche Phänomene, die keine direkten körperlichen Auswirkungen hatten, die aber deshalb als nicht weniger gefährlich wahrgenommen wurden.

\section{Gewalt und Repräsentation / Die Medialität von Gewalt}

Gewalt funktioniert also durchaus nicht ausschliesslich als körperliche. Beschimpfungen und Beleidigungen können ebenso als gewaltsam erlebt werden wie Vorstellungen. In der jüngeren Forschung wird das Sprechen selbst unter bestimmten Bedingungen als Gewalt-Handlung konzipiert. In Anlehnung an Judith Butlers Excitable Speech, in dem sich die Autorin den gewaltförmigen und verletzenden Potentialen von Sprache widmet ${ }^{40}$, erproben Literatur- und Geschichtswissenschaft der Vormoderne einen Begriff von Gewalt, der Sprache und Gewalt nicht als Gegensätze (Kultur versus Kulturverlust oder Zivilisation versus Barbarei ${ }^{41}$ ), sondern interrelational denkt. Damit wird der Performativität von Sprache (oder von Bildern) Rechnung getragen, es wird nach Wahrnehmung, Inszenierung und Funktion von Gewalthandlungen und nach ihrer besonderen sprachlichen Bedingtheit gefragt. Auch Manuel Braun und Cornelia Herberichs machen in ihrer Einleitung $\mathrm{zu}$ «Gewalt im Mittelalter» auf die soziale Bedeutung der in

39 Mommertz 2005, $344 f$.

40 Butler 1998.

41 Krämer 2005, 5. 
Texten und Bildern dargestellten Gewalt aufmerksam, weisen jedoch zugleich auf die enge Koppelung von Handlung und Kommunikation sowie darauf hin, dass «die Repräsentation von Gewalt in Texten nicht unabhängig von der Praxis der Gewalt ist $»^{42}$.

In diesem Sinn konnte Claudia Jarzebowski zeigen, dass die Repräsentation von Gewalt in einer autobiographischen Erzählung eine besondere Form der Selbstermächtigung sein kann, wenn die Autorin über das Schreiben das handlungsmächtige Potential von Sprache reflektiert und damit ihre eigene Position in einem machtpolitischen Gewaltgefüge (um)definieren $\mathrm{kann}^{43}$.

An Märtyrerlegenden, denen eine besonders drastische Gewalt-Metaphorik eignet, hat Elke Koch herausgearbeitet, dass die Gewaltdarstellung nicht allein auf die Materialität des (Heiligen-)Körpers zielt, sondern auf die sprachliche Inszenierung selbst. Eine Drohung sei zwar immer auf den Körper bezogen, jedoch auf sprachlicher Ebene. Damit seien Drohungen Schaltstellen zwischen Zeichen und Körper und gestatteten, verschiedene Ebenen des Gewaltvollzugs zu charakterisieren: Gewalt werde als, über und durch Sprache repräsentiert, auch wenn ihr Bezugspunkt immer der Körper bleibe ${ }^{44}$.

Mireille Schnyder macht an Beispielen der hoch- und spätmittelalterlichen Literatur deutlich, dass Gewalt im höfischen Roman zwingend Gewalt der Sprache ist. Als erzählte Gewalt ist sie zugleich auch immer schon gedeutete Gewalt. Es ist die sprachliche Realisierung selbst, die Gewalt herstellt - aber als Erzählung eben auch eingrenzen kann $^{45}$. Wie auf der Handlungsebene auch hat Gewalt in Texten eine bedeutungskonstituierende Wirkung, die zwischen Kommunikation, Verhandlung und zerstörerischer Anwendung changieren kann.

Diese oszillierende Qualität von Gewalt ist es, die ihre Wirkungsmacht ausmacht. Die Betrachtung von Gewaltphänomenen, wie sie in aktuellen Bildern, aber auch in frühneuzeitlichen festgehalten sind, ist immer auch mit einem Faszinosum besetzt. Gewaltdarstellungen provozieren im Betrachter eine besonders starke Reaktion: im Regime der mimetischen Ähnlichkeit ist die Gewalt, die ein anderer Körper erfährt, potentiell immer auch eine Bedrohung für den eigenen Körper. Doch im Moment des Blickens auf den anderen sind Verletzungen und Blut, ist der Horror der Einfühlung auch mit voyeuristischer Lust besetzt.

42 Braun/Herberichs 2005, 18.

43 Jarzebowski 2007.

44 Koch 2007.

45 Schnyder 2005. 
Rolf Sieferle hat die Ähnlichkeit der Wirkung von pornographischem Bild und Gewaltbild unterstrichen und gefragt: «verstricken wir uns [...] nicht ähnlich wie bei der Pornographie in eine paradoxe Faszination, eine lustbesetzte Abwehr?» ${ }^{46}$ Abscheu und Faszination sind im Gewaltbild eng miteinander verbunden und garantieren eine extreme Aufmerksamkeit des Betrachters.

Gewaltdarstellungen richten sich direkt an die Emotionen des Betrachters, sie rufen unmittelbare Affekte hervor, die das Bild an die Stelle von «Wirklichkeit» setzen. Die Betrachter derart emotional zu involvieren ist eine ästhetische Strategie der Darstellung von Gewalt, in der der Anblick inszenierter Grausamkeit Betroffenheit produziert, die die Wahrnehmung auf den Gewaltakt konzentriert. Dabei werden die Fragen nach der Funktion der Darstellungen ausgeblendet, die jedoch weitere Bedeutungsdimensionen erschliessen würden: «Violence is [...] a hermeneutical script for the exploration of meanings that reside elsewhere: scenes of torture point beyond themselves to the production of prejudice, doctrine, and [...] real violence, directed against the pagan past, women, Muslims, Jews and denigrated social classes», schreibt Robert Mills im Rahmen einer Analyse von Passionsikonographie im Mittelalter ${ }^{47}$. Das Bild weist also über sich selbst hinaus, ist eingebunden in soziales und kulturelles Handeln. Auf einer ästhetischen Ebene verfolgt Silke Tammen die Darstellung gemarterter Körper als Momente der Wahrnehmungssteuerung und der Herstellung von Lesbarkeit. Sie interpretiert die deutlich sichtbare Künstlichkeit (spät)mittelalterlicher Gewaltdarstellungen als Strategie der «Zähmung» von Gewalt, so dass herkömmliche Einschätzungen der Bilder als Darstellungen unmittelbarer Gewalthaftigkeit überholt erscheinen ${ }^{48}$.

An die ästhetische Bedeutung der Gewaltdarstellung ist eine weitere Facette des vormodernen Gewaltbegriffs gebunden, die in der berühmten Formulierung Dürers «Dann der gewalt der kunst [...] meystert alle werck» enthalten ist. Hier ist «gewalt» einerseits die Fähigkeit oder das Vermögen des Künstlers, das, was er in der Wirklichkeit vor Augen hat, in die künstliche Flächigkeit des Bildes zu überführen. Andererseits ist die «gewalt» der Kunst genau diese Überführung von Leben in Kunst und damit in ein ästhetisches Ordnungssystem.

Auf verschiedenen Ebenen operiert die Kunst mit ihrer Gewaltsamkeit. Auf der Ebene der Wirkung überwältigt sie ihre Betrachter mit der Darstellung äusserster Grausamkeit. Kunst produziert einen Schockeffekt, der die

46 Sieferle/Breuninger 1998, 10.

47 Mills 2001, 207.

48 Tammen 2005. 
Betrachter direkt zum dargestellten Geschehen in Beziehung setzt ${ }^{49}$. Diese Darstellungsstrategie kennzeichnet z.B. Tizians Gemälde der Vergewaltigung der Lucrezia ${ }^{50}$ und ist dann in der theatralischen Kunst des italienischen Barock auf ihren Gipfel getrieben ${ }^{51}$.

Auf der Ebene der Repräsentation überführt Kunst Gewalt in Ordnung. So demonstrieren in der anatomischen Darstellung Knochen- und Muskelmänner in klassischen Posen verschiedene Stadien der Sektion. Hier sind die Grausamkeit der Häutungen und die Visualisierungen des geöffneten Leibes durch ihre klassifizierende Darstellung überhöht. Anatomische Lehrbilder sind Bilder der Ordnung, die den Kontext der Sektion unsichtbar machen und damit die Gewalt aus der Darstellung verbannen. In der Materialität der Farbgebung jedoch kehrt die Bedrohlichkeit des Sektionsaktes zurück. Doch in der Häutung wird nicht nur die von der Medizin ausgeübte Gewalt am menschlichen Körper thematisiert, sondern auch die Darstellungsgewalt der Kunst über den Körper ausgedrückt. Die Gewalt wird wiederum in Ästhetik überführt ${ }^{52}$.

Was all diese Untersuchungen zeigen, ist vor allem, dass es einer genauen Kontextanalyse der Gewalt - und auch ihrer Darstellung - bedarf. Die Erkenntnis, dass Gewalthandlungen eine ritualisierte Form der Kommunikation sein können, verdankt sich auch der Interpretation von Kultur als Text ${ }^{53}$. Sie gestattet uns, Handlungen als Zeichensysteme zu «lesen», auch wenn, wie im Fall der Gewalt, diese Zeichensysteme ausgesprochen blutig sein können. Gleichzeitig wird deutlich, dass Repräsentationen ihrerseits performativ sind und so Vorstellungen und Verhalten beeinflussen. Um das Funktionieren von Gewalt im einzelnen zu begreifen, sind also einerseits der kulturelle Kontext der Gewalthandlung und andererseits der ästhetischmediale Kontext der Darstellung der Gewalthandlung signifikant. Auch wenn die körperlichen Konsequenzen von Gewaltanwendung zunächst die sichtbareren sind, so sind es die Strategien der Repräsentation von Gewalt, die unsere Imagination und unser Sprechen über Gewalt strukturieren und so unser Verständnis von Gewalt formen. Die Definition von Gewalt ist historisch veränderbar und bedarf - wie Valentin Groebner ausführt einer konsequenten Historisierung ${ }^{54}$, um den «Denkraum der Besonnenheit» (Aby Warburg) auch angesichts des Schrecklichen zurückzugewinnen. Die Historisierung stellt Fragen nach dem Wer, Wann, Wo und Mit-welcher-

49 Groebner 2003, 96-105.

50 Bohde 2002, 187-192.

51 Lang 2001.

52 Bohde 2002, 301-312. Oy-Marra 2007, 264.

53 Bachmann-Medick 1998.

54 Groebner 2003, 165-174. 
Absicht. Dazu kommt die Frage nach dem Wie, die die mediale Gestalt der Darstellung betrifft. Bilder funktionieren gemäss eigener Regeln, die auch unabhängig von der Intention ihres Einsatzes zum Tragen kommen. Nur ein Bewusstsein von der medialen Konstruiertheit von Gewalt kann den faszinierten oder den moralisierenden, den wertenden oder erschrockenen Blick brechen, der auf das «Eigentliche» zu sehen glaubt, und kann so dem Bann des Bildes die Lust an der Erkenntnis entgegensetzen.

\section{Bibliographie}

Algazi, Gadi, «Sie würden nach hinten so gail.» Vom sozialen Gebrauch der Fehde im 15. Jahrhundert, in: Thomas Lindenberger/Alf Lüdtke (Hrsg.), Physische Gewalt. Studien zur Geschichte der Neuzeit (Frankfurt 1995) 39-77

- Herrengewalt und Gewalt der Herren im späten Mittelalter. Herrschaft, Gegenseitigkeit und Sprachgebrauch, Historische Studien 17 (Frankfurt 1996)

Althoff, Gerd, «Regeln der Gewaltanwendung im Mittelalter», in: Rolf Peter Sieferle/Helga Breuninger (Hrsg.), Kulturen der Gewalt. Ritualisierung und Symbolisierung von Gewalt in der Geschichte (Frankfurt 1998) 154-170

Bachmann-Medick, Doris (Hrsg.), Kultur als Text. Die anthropologische Wende in der Literaturwissenschaft (Frankfurt 1998)

Bähr, Andreas, «Die Semantik der Ungarischen Krankheit», in: Claudia Ulbrich/Claudia Jarzebowski/Michaela Hohkamp (Hrsg.), Gewalt in der Frühen Neuzeit. Beiträge zur 5. Tagung der Arbeitsgemeinschaft Frühe Neuzeit im VHD (Berlin 2005) 359-374

Bauman, Zygmunt, Moderne und Ambivalenz. Das Ende der Eindeutigkeit (Hamburg 1995)

Bohde, Daniela, Haut, Fleisch und Farbe. Körperlichkeit und Materialität in den Gemälden Tizians (Emsdetten 2002)

Bourdieu, Pierre, «La domination masculine», Actes de la recherche en sciences sociales 84 (1990) 2-31

Braun, Manuel/Cornelia Herberichs (Hrsg.), Gewalt im Mittelalter. Realitäten - Imaginationen (München 2005)

Burghartz, Susanna, Leib, Ehre und Gut. Delinquenz in Zürich Ende des 14. Jahrhunderts (Zürich 1990)

- «Hat Gewalt ein Geschlecht? Argumentationsstrategien und Verhaltensmuster in vormodernen Gesellschaften», in: Frauenrat der Universität Konstanz (Hrsg.), «Kriminalität und Geschlecht», Vortragsreihe im Wintersemester 1998/99 (Konstanz 1999) 31-45

Burschel,Peter/Götz Distelrath/Sven Lembke (Hrsg.), Das Quälen des Körpers. Eine historische Anthropologie der Folter (Köln 2000)

Butler, Judith, Hass spricht. Zur Politik des Performativen (Berlin 1998) (zuerst New York 1997)

Chaytor, Miranda, "Husband(ry): Narratives of Rape in the Seventeenth Century", Gender \& History 7/3 (1995) 378-407

Crouzet, Denis, «Die Gewalt zur Zeit der Religionskriege im Frankreich des 16. Jahrhunderts», in: Thomas Lindenberger/Alf Lüdtke (Hrsg.), Physische Gewalt. Studien zur Geschichte der Neuzeit (Frankfurt 1995) 78-105

- «Königliche und religiöse Gewalt im Massaker der Bartholomäusnacht oder der 〈Wille〉 Karls IX.», in: Claudia Ulbrich/Claudia Jarzebowski/Michaela Hohkamp (Hrsg.), Gewalt in der Frühen Neuzeit. Beiträge zur 5. Tagung der Arbeitsgemeinschaft Frühe Neuzeit im VHD (Berlin 2005) 33-58

Dinges, Martin, «Formenwandel der Gewalt in der Neuzeit. Zur Kritik der Zivilisationsheorie von Norbert Elias», in: Rolf Peter Sieferle/Helga Breuninger (Hrsg.), Kulturen der Gewalt. Ritualisierung und Symbolisierung von Gewalt in der Geschichte (Frankfurt 1998) 171-194

Elias, Norbert, Über den Prozess der Zivilisation, 2 Bde. (Basel 1939) 
Eming, Jutta/Claudia Jarzebowski (Hrsg.), Blutige Worte: Internationales und interdisziplinäres Kolloquium zum Verhältnis von Sprache und Gewalt in Mittelalter und Früher Neuzeit (Göttingen 2007)

Faro, Marlene, "An heymlichen orten». Männer und der weibliche Unterleib. Eine andere Geschichte der Gynäkologie (Leipzig 2002)

Galtung, Johan, Strukturelle Gewalt. Beiträge zur Friedens- und Konfliktforschung (Reinbek bei Hamburg 1975)

Gender \& History $16 / 3$ (Nov. 2004)

Groebner, Valentin, «Der verletzte Körper und die Stadt. Gewalttätigkeit und Gewalt in Nürnberg am Ende des 15. Jahrhunderts», in: Thomas Lindenberger/Alf Lüdtke (Hrsg.), Physische Gewalt. Studien zur Geschichte der Neuzeit (Frankfurt 1995) 162-189

- Ungestalten. Die visuelle Kultur der Gewalt im Mittelalter (München 2003)

Heitmeyer, Wilhelm/Hans-Georg Soeffner (Hrsg.), Gewalt (Frankfurt 2004)

Hilbig, Antje/Claudia Kajatan/Ingrid Miethe (Hrsg.), Frauen und Gewalt. Interdisziplinäre Untersuchungen zu geschlechtsgebundener Gewalt in Theorie und Praxis (Würzburg 2002)

Historische Anthropologie, Themenschwerpunkt: Gewalt, hg. v. Fritz W. Kramer und Alf Lüdtke, $2 / 2004$

L'homme, Gewalt, hg. v. Andrea Griesebner und Claudia Ulbrich, 7,2 (1996)

Jarzebowski, Claudia, «Gewalt und Emotion in der Frühen Neuzeit», in: Eming/Jarzebowski 2007

Koch, Elke, «Formen und Bedingungen von Sprachgewalt in Katharinenlegende und -spiel», in: Eming/Jarzebowski 2007

Krämer, Sybille, Gewalt der Sprache - Sprache der Gewalt, Hrsg. von der Landeskommission Berlin gegen Gewalt (Berlin 2005)

Lang, Walther K., Grausame Bilder: Sadismus in der neapolitanischen Malerei von Caravaggio bis Giordano (Berlin 2001)

Lindenberger, Thomas/Alf Lüdtke (Hrsg.), Physische Gewalt. Studien zur Geschichte der Neuzeit (Frankfurt 1995)

Lorenz, Maren, Leibhaftige Vergangenheit. Einführung in die Körpergeschichte (Tübingen 2000)

Martus, Steffen/Marina Münkler/Werner Röcke (Hrsg.), Schlachtfelder. Codierung von Gewalt im medialen Wandel (Berlin 2003)

Meumann, Markus/Dirk Niefanger (Hrsg.), Ein Schauplatz herber Angst. Wahrnehmung und Darstellung von Gewalt im 17. Jh. (Göttingen 1997)

Mills, Robert, "Violence in Medieval Passion Iconography", Fifteenth-Century Studies 27 (2001) 200-215

Mommertz, Monika, «Imaginative Gewalt». Praxe(m)ologische Überlegungen zu einer vernachlässigten Gewaltform», in: Ulbrich/Jarzebowski/Hohkamp 2005, 343-357

Morris, David B., Geschichte des Schmerzes, aus dem Amerikanischen von Ursula Gräfe (Frankfurt 1994) (zuerst 1991)

Nolde, Dorothea, Gattenmord. Macht und Gewalt in der frühneuzeitlichen Ehe (Köln 2003)

Nunner-Winkler, Gertrud, «Überlegungen zum Gewaltbegriff», in: Wilhelm Heitmeyer/HansGeorg Soeffner (Hrsg.), Gewalt (Frankfurt 2004) 21-61

Oy-Marra, Elisabeth, «Bildstrategien von Schrecken und Erlösung. Der geschundene Körper christlicher Märtyrer in der Malerei des 16. und 17. Jahrhunderts», in: Martin Zenck/Tim Becker/Raphael Woebs (Hrsg.), Gewaltdarstellung und Darstellungsgewalt in den Künsten und Medien (Berlin 2007) 249-273

Porret, Michel (Hrsg.), Le corps violenté: du geste à la parole (Genève 1998)

Rath, Brigitte, ««... und wolt das Schert durch in stossen.> Zur physischen Gewalt in Südtirol um 1500», L'Homme 2 (1996) 56-69

Rey, Roselyne, Histoire de la douleur (Paris 1993)

Scarry, Elaine, Der Körper im Schmerz. Die Chiffren der Verletzlichkeit und die Erfindung der Kultur, aus dem Amerikanischen von Michael Bischoff (Frankfurt 1992) (zuerst 1985)

Schnyder, Mireille, «Erzählte Gewalt und die Gewalt des Erzählens. Gewalt im deutschen höfischen Roman», in: Braun/Herberichs 2005, 365-379

Schwerhoff, Gerd, Aktenkundig und gerichtsnotorisch. Einführung in die historische Kriminalitätsforschung (Tübingen 1999)

- «Zivilisationsprozess und Geschichtswissenschaft. Norbert Elias' Forschungsparadigma in historischer Sicht», Historische Zeitschrift 266 (1998) 561-605 
Sieferle, Rolf Peter/Helga Breuninger (Hrsg.), Kulturen der Gewalt. Ritualisierung und Symbolisierung von Gewalt in der Geschichte (Frankfurt 1998)

Sofsky, Wolfgang, Traktat über die Gewalt (Frankfurt 1996)

- Zeiten des Schreckens. Amok, Terror, Krieg (Frankfurt 2002)

Spierenburg, Peter (Hrsg.), Men and Violence: Gender, Honor, and Rituals in Modern Europe and America (Columbus 1998)

Tammen, Silke, «Gewalt im Bilde: Ikonographien, Wahrnehmungen, Ästhetisierungen», in: Manuel Braun/Cornelia Herberichs (Hrsg.), Gewalt im Mittelalter. Realitäten-Imaginationen (München 2005) 307-340

Traverse, Gewalt - Violence, 1/1995

Ulbrich, Claudia/Claudia Jarzebowski/Michaela Hohkamp (Hrsg.), Gewalt in der Frühen Neuzeit. Beiträge zur 5. Tagung der Arbeitsgemeinschaft Frühe Neuzeit im VHD (Berlin 2005)

Zemon Davis, Natalie, «Riten der Gewalt», in: Natalie Zemon Davis, Humanismus, Narrenherrschaft und die Riten der Gewalt: Gesellschaft und Kultur im frühneuzeitlichen Frankreich, aus dem Amerikanischen von Nele Löw Beer, mit einem Nachwort von Norbert Schindler (Frankfurt 1987) 171-209 (zuerst 1975) 